\title{
The near-infrared companion to HD 94660 (=KQ Vel)
}

\author{
M. Schöller ${ }^{1}$, C. A. Hummel ${ }^{1}$, S. Hubrig ${ }^{2}$, D. W. Kurtz ${ }^{3,4}$, G. Mathys ${ }^{5}$, J. Robrade ${ }^{6}$, and S. P. Järvinen ${ }^{2}$ \\ 1 European Southern Observatory, Karl-Schwarzschild-Str. 2, 85748 Garching, Germany \\ e-mail: mschoell@eso.org \\ 2 Leibniz-Institut für Astrophysik Potsdam (AIP), An der Sternwarte 16, 14482 Potsdam, Germany \\ 3 Centre for Space Research, Physics Department, North West University, Mahikeng 2745, South Africa \\ 4 Jeremiah Horrocks Institute, University of Central Lancashire, Preston PR1 2HE, UK \\ 5 European Southern Observatory, Alonso de Cordova 3107, Vitacura, Santiago, Chile \\ ${ }^{6}$ Hamburger Sternwarte, Gojenbergsweg 112, 21029 Hamburg, Germany
}

Received 16 July 2020 / Accepted 10 September 2020

\begin{abstract}
Context. The Bp star HD 94660 is a single-lined spectroscopic binary. Some authors have suggested that the unseen companion of at least $2 M_{\odot}$ may be a compact object.

Aims. We intend to study this multiple system in detail, especially to learn more about the thus far unseen companion.

Methods. We have collected and analyzed PIONIER (Precision Integrated-Optics Near-infrared Imaging ExpeRiment) $H$-band data from the Very Large Telescope Interferometer, TESS (Transiting Exoplanet Survey Satellite) visible photometric data, and X-ray observations with Chandra of HD 94660.

Results. Using PIONIER, we were able to detect the companion to HD 94660, which is absent from high quality spectra at visible wavelengths, with a magnitude difference of 1.8 in the $H$ band at a separation of 18.72 mas. The TESS light curve shows variations with a period of $2.1 \mathrm{~d}$ and also flaring. The Chandra spectrum is well described by emission from hot thermal plasma, yet it might include a nonthermal component. The X-ray properties are compatible with a magnetically active companion, while it is also possible that there might be some magnetospheric contributions from the primary.

Conclusions. We can rule out that the companion to HD 94660 is a compact source. It is also very unlikely that this companion is a single star, as the estimated mass of more than $2 M_{\odot}$, the magnitude difference of 1.8 in the $H$ band, and its nondetection in visible spectra are difficult to realize in a single object. One alternative could be a pair of late F stars, which would also be responsible for the detected photometric variations. Interferometric observations over the full binary orbit are necessary to determine the real mass of the companion and to add constraints on the overall geometry of the system.
\end{abstract}

Key words. binaries: close - stars: chemically peculiar - techniques: interferometric - techniques: photometric - X-rays: stars

\section{Introduction}

HD $94660(=\mathrm{KQ} \mathrm{Vel})$ is a rather bright $\left(m_{V}=6.11\right)$, chemically peculiar Bp star (Renson \& Manfroid 2009 list a spectral type of A0p EuCrSi) with a strong magnetic field, which Bailey et al. (2015) characterize with a magnetic dipole strength of $B_{\mathrm{d}}=$ $7500 \mathrm{G}$ with additional quadrupole and octupole moments, an inclination angle of the stellar rotation axis of $i=16^{\circ}$, and a magnetic obliquity angle of $\beta=30^{\circ}$. As its measured mean longitudinal magnetic field on the order of $-2 \mathrm{kG}$ is almost constant, HD 94660 is frequently used as a magnetic standard star. It also shows spectral lines resolved into their magnetically split components.

Globally ordered magnetic fields are observed in roughly $10 \%$ of the intermediate and massive main-sequence stars with spectral types between approximately B2 and F0. These stars, which are generally called the chemically peculiar Ap and Bp stars (or Ap/Bp stars), exhibit strong overabundances of certain elements, such as iron peak elements and rare earths, and underabundances of $\mathrm{He}, \mathrm{C}$, and $\mathrm{O}$, relative to solar abundances. Massive Bp stars usually show overabundances of $\mathrm{He}$ and $\mathrm{Si}$. Ap/Bp stars show strict periodic light, spectral, and magnetic variations with the rotation period, which are well understood in terms of the oblique rotator model, in which the magnetic axis is inclined with respect to the rotation axis (Stibbs 1950).
HD 94660 has an effective temperature of $T_{\text {eff }}=11300 \pm$ $400 \mathrm{~K}$, a mass of $3.0 \pm 0.2 M_{\odot}$ (Bailey et al. 2015), and a rotation period of $2800 \pm 200 \mathrm{~d}$ (Mathys 2017). A temperature of $11300 \mathrm{~K}$ is indicative of a spectral type B8.5p (e.g., Eker et al. 2020), rather than the A0p indicated by Renson \& Manfroid (2009). Hensberge (1993) already reported long-term photometric variability with peak-to-peak amplitudes of $\sim 0.03 \mathrm{mag}$ and determined a period on the order of $2700 \mathrm{~d}$. Bailey et al. (2015) estimated that HD 94660 has completed less than half of its main sequence lifetime. Its parallax is $8.75 \pm 0.16$ mas (Gaia Collaboration 2018). Mathys et al. (1997) reported variability of the radial velocity that is indicative of a spectroscopic binary with an orbital period somewhat longer than two years. These variations, with a total range of $35 \mathrm{~km} \mathrm{~s}^{-1}$, were confirmed by Bailey et al. (2015) using UVES (UV-visual echelle spectrograph), HARPSpol (High Accuracy Radial velocity Planet Searcher polarimeter), and ESPaDOnS (Echelle SpectroPolarimetric Device for the Observation of Stars) spectra. These authors report that the most likely period of these variations is on the order of $840 \mathrm{~d}$. The most recent orbital elements of the system were derived by Mathys (2017) with an orbital period of $P_{\text {orb }}=848.96 \pm 0.13 \mathrm{~d}$, an eccentricity of $e=0.4476 \pm 0.0049$, and a mass function of $f(M)=0.3631 \pm 0.0075 M_{\odot}$. According to the mass function, the secondary must be rather massive $\left(M>2 M_{\odot}\right)$, yet there is no hint of it in visible spectra. This led 
Bailey et al. (2015) to the conclusion that the companion might be a compact object, such as a neutron star, a black hole, or a pair of white dwarfs.

While about two thirds of the Galactic massive stars appear to be members of close binary systems (Sana et al. 2012), the study of Carrier et al. (2002) indicated a scarcity of magnetic $\mathrm{Ap} / \mathrm{Bp}$ stars in close binaries. On the other hand, the occurrence of $\mathrm{Ap} / \mathrm{Bp}$ stars in wide binaries is relatively high. In the most recent study of $43 \mathrm{Ap} / \mathrm{Bp}$ stars with magnetically resolved lines by Mathys (2017), 22 stars are binaries, with a shortest orbital period $P_{\text {orb }}$ of those systems of $27 \mathrm{~d}$. The observed dearth of magnetic $\mathrm{Ap} / \mathrm{Bp}$ stars in close binaries is probably related to the origin of the magnetic fields in the Ap/Bp stars. Recently proposed scenarios suggest that these stars result from the merging of two lower mass stars or protostars (Tutukov \& Fedorova 2010; Ferrario et al. 2009). The merged star would undergo a brief period of strong differential rotation, which gives rise to large-scale magnetic fields in the radiative envelope.

To better understand the nature of the companion, we carried out interferometric observations of HD 94660 with PIONIER (Precision Integrated-Optics Near-infrared Imaging ExpeRiment) on the Very Large Telescope Interferometer (VLTI) within the framework of our PIONIER Bp multiplicity survey, looking for wider companions that could have helped an inner binary pair to merge. Further, we analyzed TESS (Transiting Exoplanet Survey Satellite) photometric observations and Chandra X-ray data. Finally, we discuss the results of our multiwavelength observations of this system.

\section{Interferometric observations}

We obtained one observation of HD 94660 with the PIONIER beam combining instrument (Le Bouquin et al. 2011) on the VLTI (e.g., Schöller 2007) on 2018 May 18. PIONIER combines four beams in the $H$-band, either from the $8.2 \mathrm{~m}$ Unit Telescopes (UTs), or, as in our case, from the $1.8 \mathrm{~m}$ Auxiliary Telescopes (ATs), which leads to visibilities on six different baselines as well as four closure phase measurements, simultaneously. We used PIONIER's low resolution spectroscopic optics to measure within the $H$-band at five different wavelengths, thus improving the $u v$-coverage. Alternatively, the instrument can also operate in integrated light for sensitivity enhancement on faint targets.

All PIONIER data were reduced by us with the pndrs pipeline (Le Bouquin et al. 2011). It uses the diameters listed in the JSDC catalog (Chelli et al. 2016) for the interferometric calibrator star observed before and after the science target to compute the transfer function used to calibrate the squared visibility amplitudes and the closure phases of the science target. In our case, HD 95370, with a diameter of 0.52 mas (Lafrasse et al. 2010), served as the calibrator. HD 95370 is not listed in the bad calibrator (BadCal) list ${ }^{1}$.

We used the Python tool CANDID ${ }^{2}$ (Companion Analysis and Non-Detection in Interferometric Data) developed by Gallenne et al. (2015) to search for a companion in the separation range of up to 40 mas (limited by bandwidth smearing). CANDID performs a search on an adaptive grid with a spacing that allows it to find the global minimum in $\chi^{2}$. In order to assess the significance of a companion detection, not only is the reduced $\chi^{2}$ considered, but the number of degrees of freedom is as well. To support a more stable search with CANDID, we $\begin{array}{ll}1 & \text { www. jmmc. fr/badcal/show. jsp?type=all\&display=simple } \\ 2 & \text { github.com/amerand/CANDID. }\end{array}$
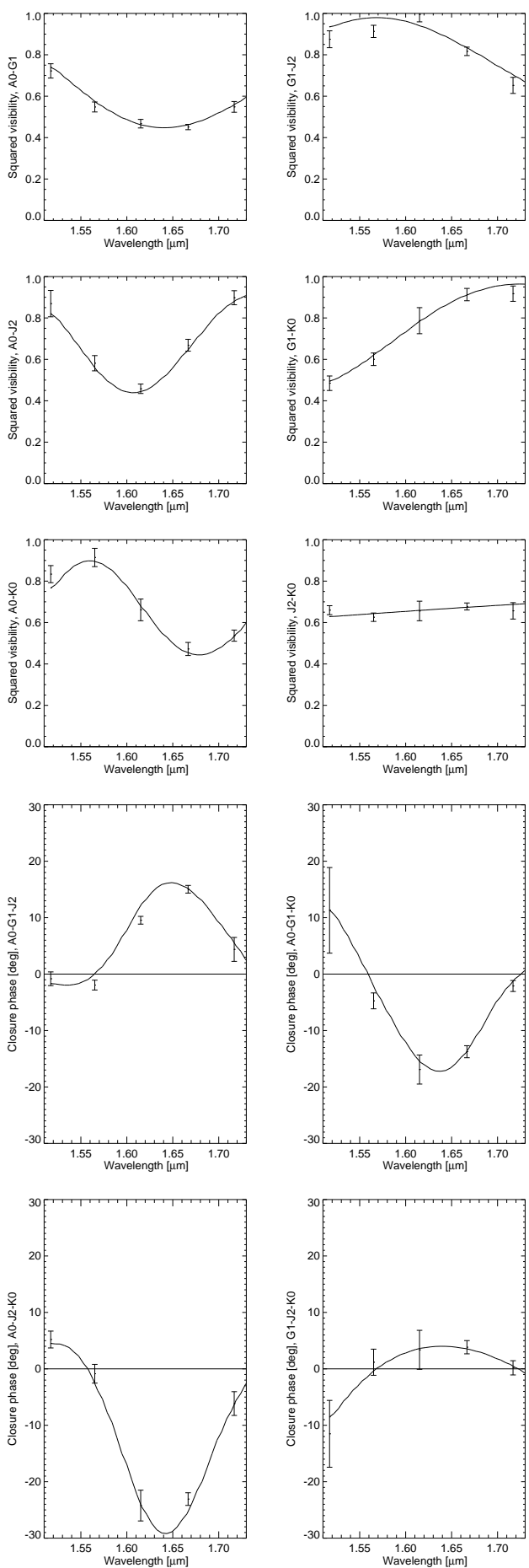

Fig. 1. Six visibilities (top) and four closure phases (bottom) of the first PIONIER observations of HD 94660. The curves are the binary fit to the data.

constrained the diameter of the (primary) star to be equal to its surface brightness diameter (Mozurkewich et al. 2003).

We detect a companion to HD 94660 with an $H$ band magnitude difference of $1.8 \pm 0.03$ at a separation of $18.72 \pm 0.02$ mas and a position angle of $262.74^{\circ} \pm 0.05^{\circ}$ (east of north). The error ellipse, following the CLEAN beam, is $0.07 \times 0.03$ mas, with a position angle of $6^{\circ}$. The binary fit to the data is highly convincing, with no hints for further components (see Fig. 1).

The angular separation corresponds to a projected linear separation of slightly more than 2 au, given HD 94660 's parallax 

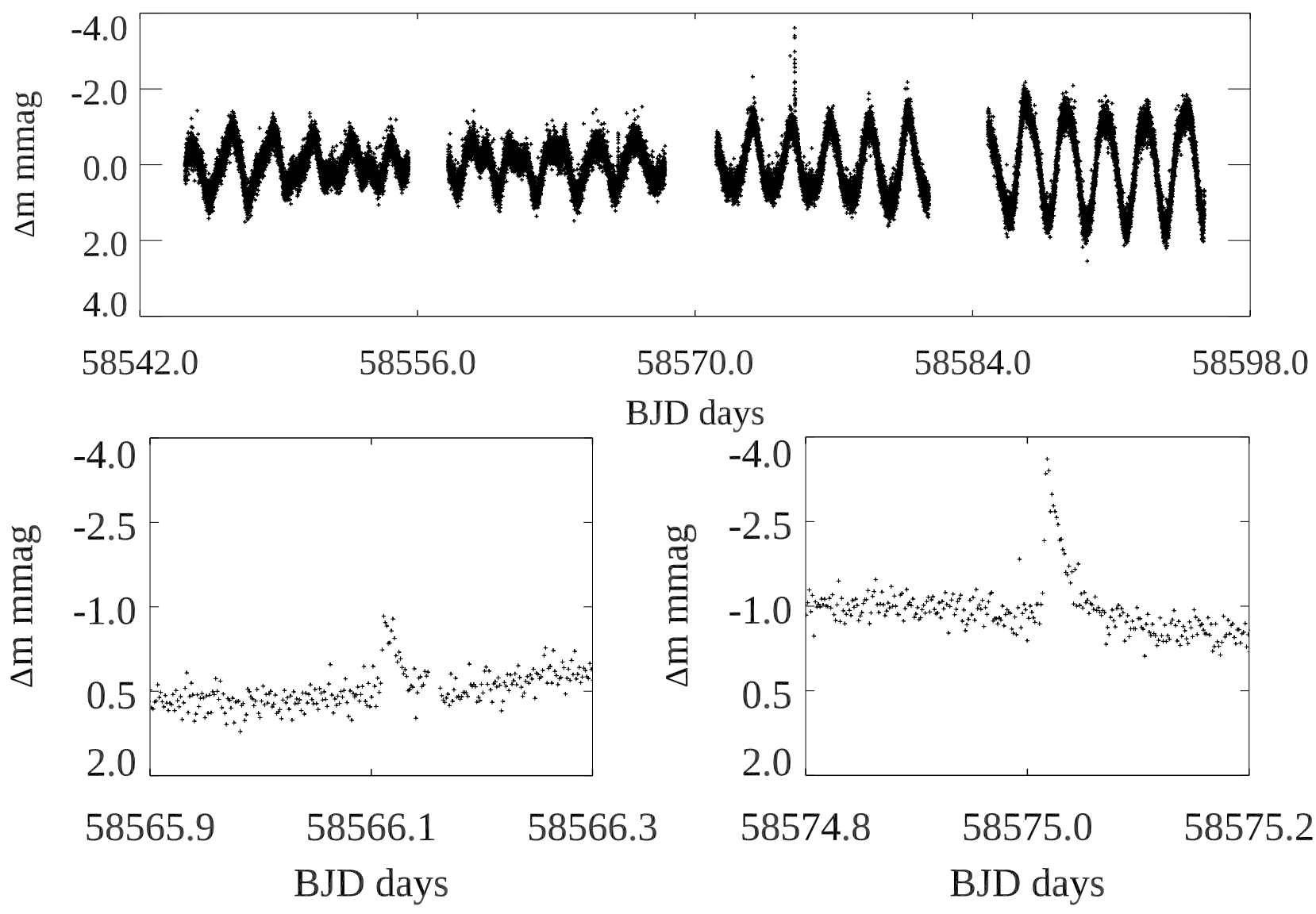

Fig. 2. Top: full Sectors 9 and 10 light curve of TIC 147622676 showing variations indicative of rotation in a cool, spotted star. Bottom: two flares seen after a highpass filter removed much of the low frequency rotational and instrumental variations. The flares are also indicative of a cool, active star. The times are bolocentric Julian date (BJD)-2400000.0.

of 8.75 mas (Gaia Collaboration 2018). Depending on the total mass of the system (we used $5 M_{\odot}$ ) and the real linear size of the orbit, this points to an orbital period of at least $1.4 \mathrm{yr}$, which is of the same magnitude as the orbital period of $849 \mathrm{~d}$ found by Mathys (2017). Therefore, we conclude that we indeed have found the thus far unseen spectroscopic companion with PIONIER. The $H$ band magnitude difference is not consistent with a compact companion.

\section{TESS photometric observations}

TESS observations of HD 94660 (TIC 147622676) were obtained in Sectors 9 and 10 in 120 s cadence, as is seen in the top panel of Fig. 2. Those observations show significant periodic variations on a time scale of $2.1 \mathrm{~d}$. These are variable in both shape and amplitude, as is characteristic of cool stars that show rotational variation from time-variable spots.

There are two clear instances of flares in the TESS data. These are easier to see with the rotational and some instrumental variations filtered from that data, as is shown in the bottom panels of Fig. 2. Both flares show a typical fast rise and then decay on a time scale of about 30-60 min. The observed amplitudes are only $\sim 1$ and $2 \mathrm{mmag}$, but the intrinsic amplitudes are much larger when taking the brighter primary component of HD 94660 into account.

If we take an F8V star as an example of a cooler flare star with variable spots, then the absolute magnitude difference between this star and the primary B8.5p star is about 4 . Thus, we find that the supposed F8V star contributes about $2.5 \%$ of the light of the system. Scaling up by the inverse of that suggests the flares on the cool star have amplitudes on the order of 0.05 mag, which is reasonable for these types of stars. We did not include any bolometric correction or possible extinction, since this is a rough estimate in order to show the scale of the flares.

Clearly, the Bp star cannot be responsible for the $2.1 \mathrm{~d}$ photometric variations; Ap/Bp stars have stable spots with a constant amplitude and shape rotational light curves. A chance alignment with an unrelated source is also very unlikely, as no suitable source is known in the sparsely populated field.

\section{X-ray emission}

X-ray observations of HD 94660 were obtained by the Chandra observatory with ACIS-I (Advanced CCD Imaging Spectrometer) on 2016 August 20 with an exposure time of 25 ks (ObsID: 17745). The Chandra data processing was done with the CIAO (Chandra Interactive Analysis of Observations) 4.10 software package and its standard tools were used to produce light curves and spectra. Source photons were extracted from a circular region with a $5^{\prime \prime}$ radius; the background was taken from a nearby source free region. The selected energy range is $0.3-10.0 \mathrm{keV}$ for our source products.

The target only shows low variability in the X-ray light curve, as is presented in Fig. 3. Thus, we used all of the data combined for the spectral analysis, which was carried out with XSPEC $^{3}$ (X-ray Spectral Fitting Package) V12.9. We used

\footnotetext{
3 heasarc.gsfc.nasa.gov/xanadu/xspec/
} 


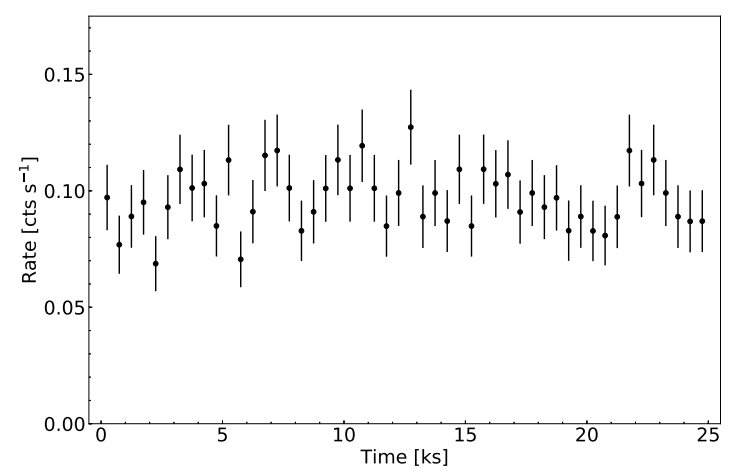

Fig. 3. X-ray light curve of HD 94660 from Chandra data, binned to $500 \mathrm{~s}$ intervals.

two-temperature plasma models based on APEC (Smith et al. 2001), which is referred to as 2APEC in the following, as well as an APEC plus powerlaw model, hereafter called APEC + POW, to fit the X-ray spectrum. The spectrum was rebinned for modeling, so that the errors are $1 \sigma$. Other models, such as APEC plus black body radiation, were tested; however, they did not yield meaningful results.

A potential absorption column and the metallicity were treated as free parameters in the initial modeling. However, the absorption $\left(n_{\mathrm{H}}\right)$ was found to be consistent with zero and therefore neglected in the further modeling. The metallicity was poorly determined and we adopted the best fit value of 0.4 solar, relative to values given by Grevesse \& Sauval (1998). Adding further model components or free parameters, such as the individual element abundances, did not lead to constrained parameters and did not significantly improve the fit. We adopted the Gaia distance of $115 \mathrm{pc}$.

The 2APEC and APEC+POW models lead to a very similar fit quality, as is shown in Fig. 4. All of the fit parameters are given in Table 1. Independent of the model details, we find a flux value of $F_{\mathrm{X}}=1.4 \pm 0.1 \times 10^{-12} \mathrm{erg} \mathrm{cm}^{-2} \mathrm{~s}^{-1}$ in the $0.3-10.0 \mathrm{keV}$ band. Overall, our analysis is in agreement, within the error bars, with the one presented by Oskinova et al. (2020) from the same data, making an allowance for the solar abundances adopted by these authors.

In the thermal case, the X-ray emission can be explained by active stars well. Its origin would be coronal magnetic activity, generated by fast rotating "normal" main-sequence stars. From the observed X-ray luminosity of $\log L_{X}=30.3$ and the coronal temperature components of $10 \mathrm{MK}$ and $25 \mathrm{MK}$ (average $T_{\mathrm{X}} \approx 20 \mathrm{MK}$ ), an active binary consisting of late-F to early Gtype stars would be a valid candidate. These spectral types are required since $\mathrm{X}$-rays from magnetic activity saturate at about $\log L_{\mathrm{X}} / L_{\mathrm{bol}} \approx-3$ (see, e.g., Güdel 2004 for an overview).

Other scenarios that are consistent with the observed X-ray properties are a Herbig Ae/Be star (e.g., Stelzer et al. 2009) or an RS CVn system (Güdel 2004). However, the required minimum mass and the observed $H$ band flux ratio are harder to reconcile with these source classes.

Magnetic Ap/Bp stars are also X-ray emitters via magnetically confined wind shocks (MCWS; Babel \& Montmerle 1997) and their magnetosphere might even generate nonthermal $\mathrm{X}$-rays via auroral emission (Leto et al. 2017). An application of this model to the A0p star CU Vir is presented in Robrade et al. (2018) and while its spectral X-ray characteristics are comparable to those of HD 94660, its X-ray luminosity is about two orders of magnitudes lower. Although the magnetospheres of
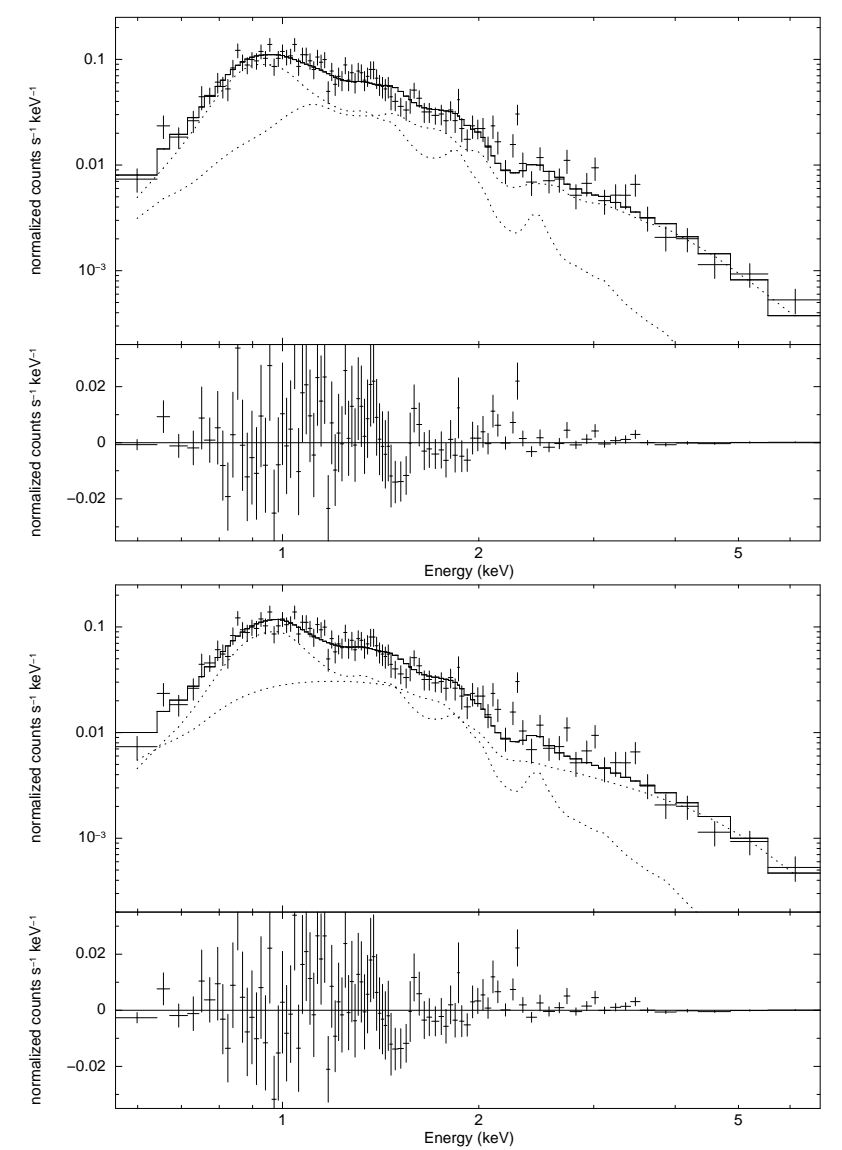

Fig. 4. X-ray spectrum of HD 94660 together with our best fit twotemperature thermal model (2APEC, top) and thermal plus nonthermal model (APEC+POW, bottom). Each plot shows the data, the model (solid line), the two components (dotted lines), and the residuals (in the subpanel).

the two stars differ due to the higher magnetic field and the slower rotation of HD 94660, $\log L_{\mathrm{X}}>30$ is above what is typically observed for comparable stars. Thus, the primary B8.5p star might very well contribute to the observed X-ray emission, but it is unlikely that it is the sole X-ray source in the system.

\section{Discussion}

The presence of a compact companion to HD 94660 is inconsistent with our detection of this companion with PIONIER on the VLTI with an $H$ band magnitude difference of 1.8. A compact companion would not be the source of the flares seen by TESS and is not required to explain the X-ray emission from HD 94660.

The Gaia parallax of HD 94660 is larger than the one determined by HIPPARcos (HIgh Precision PARallax COllecting Satellite; $8.75 \pm 0.16$ mas vs. $6.67 \pm 0.80$ mas; ESA 1997). Bailey et al. (2015) used the HIPPARCos parallax to calculate a distance of $150 \mathrm{pc}$ to HD 94660 . Using the Gaia parallax, this would lead to $115 \mathrm{pc}$, the luminosity would drop down from $105 L_{\odot}$ to $62 L_{\odot}$, and the mass would decrease from $3.0 M_{\odot}$ to $2.6 M_{\odot}$. The lower mass is, however, not in agreement with the temperature of $11300 \mathrm{~K}$, which was determined by Bailey et al. (2015). This might be a sign that current Gaia parallaxes are not reliable for bright stars or for binary objects. A full interferometric and spectroscopic orbital solution of HD 94660 would resolve this discrepancy. But even if we use this lower primary 
Table 1. X-ray spectral fit results for HD 94660.

\begin{tabular}{|c|c|c|}
\hline \multicolumn{3}{|c|}{ 2APEC } \\
\hline$k T_{1}$ & $0.84_{-0.03}^{+0.07}$ & $\mathrm{keV}$ \\
\hline $\mathrm{EM}_{1}$ & $8.52_{-0.54}^{+0.54}$ & $10^{52} \mathrm{~cm}^{-3}$ \\
\hline$k T_{2}$ & $2.43_{-0.22}^{+0.26}$ & $\mathrm{keV}$ \\
\hline $\mathrm{EM}_{2}$ & $8.20_{-0.76}^{+0.76}$ & $10^{52} \mathrm{~cm}^{-3}$ \\
\hline Abundance & 0.4 & Solar \\
\hline$\chi_{\text {red }}^{2}$ (d.o.f.) & $1.18(93)$ & \\
\hline$L_{\mathrm{X}}(0.3-10.0 \mathrm{keV})$ & 2.0 & $10^{30} \mathrm{erg} \mathrm{s}^{-1}$ \\
\hline \multicolumn{3}{|c|}{ APEC+POW } \\
\hline$k T_{1}$ & $0.94_{-0.03}^{+0.03}$ & $\mathrm{keV}$ \\
\hline $\mathrm{EM}_{1}$ & $8.63_{-0.83}^{+0.83}$ & $10^{52} \mathrm{~cm}^{-3}$ \\
\hline Abundance & 0.4 & Solar \\
\hline$\alpha$ & $2.39_{-013}^{+0.12}$ & [PhoIndex] \\
\hline Norm (at $1 \mathrm{keV}$ ) & $1.68_{-0.26}^{+0.28}$ & $10^{-4} \mathrm{ph} \mathrm{keV}^{-1} \mathrm{~cm}^{-2}$ \\
\hline$\chi^{2}$ (d.o.f.) & $1.20(93)$ & \\
\hline$L_{\mathrm{X}}(0.3-10.0 \mathrm{keV})$ & 2.3 & $10^{30} \mathrm{erg} \mathrm{s}^{-1}$ \\
\hline
\end{tabular}

mass of $2.6 M_{\odot}$ in the system's mass function, the mass of the companion would still be above $2.0 M_{\odot}$.

The first interferometric observation, which was carried out in the framework of our PIONIER Bp star multiplicity survey, showed that HD 94660 is a binary with a separation of 18.72 mas. The binary fit to the data shows no hints of further companions. The angular separation that was found corresponds to a projected linear separation of slightly more than 2 au. This points to an orbital period of at least $1.5 \mathrm{yr}$, which is of the same magnitude as the orbital period of $849 \mathrm{~d}$ found by Mathys (2017). Therefore, we conclude that we indeed have found the thus far unseen spectroscopic companion with PIONIER. We checked, once more, the spectra from UVES and HARPSpol used by Bailey et al. (2015) and could not find any traces of a companion.

HD 94660 was also observed by TESS. For extremely slowly rotating Ap/Bp stars, TESS observations generally show a flat light curve with no signal above the noise level in the Fourier transform. However, for HD 94660, TESS observed variations of a significant amplitude (several mmag peak-to-peak), which are cyclic on a timescale on the order of $2.1 \mathrm{~d}$, but not periodic, as there are considerable differences from cycle to cycle, with occasional flare-like events that are superimposed. Clearly, the primary $\mathrm{Bp}$ star cannot be responsible for the photometric variations. A chance alignment with an unrelated source is very unlikely since no suitable source is known in the sparsely populated field. The variations are very reminiscent of those of active late-type stars or pre-main sequence stars.

Our X-ray observation with Chandra shows slight flux variations over the total exposure of about $7 \mathrm{~h}$, but no major flaring. The spectral properties are compatible with magnetic activity in the companion and/or a modified magnetically confined wind shock model.

If we try to reconcile all information that we have gathered in our study of HD 94660, we would have to keep the following in mind: (a) the $H$ band magnitude difference of 1.8 between primary and secondary, (b) that the companion is not seen in spectra taken at visible wavelengths, (c) the companion mass is of at least $2 M_{\odot}$, (d) that we need a source of photometric variability with a $2.1 \mathrm{~d}$ period in the system, and (e) that we need a source for flares in the system. The primary Bp star is not the source of either the photometric variability or the flares.

If we assume that the secondary is a single main-sequence star, then the mass of at least $2 M_{\odot}$ would translate into a spectral type earlier than $\mathrm{A} 5 \mathrm{~V}$. The magnitude difference in the visible between the primary and secondary would then be on the order of 1 , which is in conflict with the companion not having been seen in the visible spectra. On the other hand, a main sequence single star that obeys the $\Delta m_{\mathrm{H}}=1.8$ would correspond to an F5V star, which can be ruled out as well since its mass is too low, around $1.5 M_{\odot}$. A $2 M_{\odot}$ giant would again be too bright in the visible and the evolutionary tracks of both components would be difficult to align. A pre-main sequence Herbig $\mathrm{Ae} / \mathrm{Be}$ star would fit the mass, but it should be of a brightness that is similar to the primary in the visible. Using the VizieR Photometry viewer ${ }^{4}$, we did not see any infrared excess for HD 94660, making this scenario quite unlikely. Also, while we expect photometric variations from a Herbig $\mathrm{Ae} / \mathrm{Be}$ star, mainly due to the accretion process, these would rather be stochastic than quasiperiodic.

If we go toward a higher order multiple system, then two fast rotating, young and active F8V stars in a close binary would together have a mass that is higher than $2 M_{\odot}$. Schmidt-Kaler (1982) gave an absolute visual magnitude of -0.25 for a B8V star and +0.2 for a B9V star. For our B8.5p star, we adopt an absolute visual magnitude of 0.0. According to Schmidt-Kaler, an F8V star has an absolute visual magnitude of +4.0. Thus, the magnitude difference between one F8 star and the late B primary in the visible would be around 4 . Given the high quality of some of the spectra of HD 94660 that have been analyzed by both Bailey et al. (2015) and by us, one can very conservatively assume that any line of the secondary that has a depth greater than $1 \%$ of the continuum should have been detected. With a flux ratio of 40 between the Bp star and one of the two F8V companions, the corresponding upper limit on the intrinsic depth of a line in the spectrum of the latter is $40 \%$ of the continuum. It is not easy to make a quantitative estimate, but it should not require such an F8V star to have a very large $v \sin i$ for even its strongest lines to have intrinsic depths of less than $40 \%$ (see, e.g., Fig. 6 of Slettebak et al. 1975 for an F5V template spectrum). We also have to note that it is quite common that flaring stars are found in binaries. Using the colors provided by Ducati et al. (2001), $(V-H)_{0}=-0.40$ for a B8.5V star and $(V-H)_{0}=1.06$ for an F8V star, we calculated a magnitude difference in the $H$ band between a B8.5V star and a single F8V star of 2.55 and between a B8.5V star and two F8V stars of 1.8 , which matches the magnitude difference measured by us with PIONIER.

In conclusion, a fast rotating and active F8V+F8V close binary as the companions of the Bp component of the HD 94660 system is plausible and compatible with the available observational constraints. Of course, combinations of two stars of similar spectral types can also explain the observational constraints.

To investigate the nature of the infrared, optically invisible, companion of HD 94660, additional interferometric observations are necessary. These observations, together with the spectroscopic solution, will allow for the determination of the full orbit, further constrain the nature of both components, and fix the distance toward HD 94660. The characterization of the companion will also help to understand the role of binarity for the origin of magnetism in stars with radiative envelopes.

\footnotetext{
vizier.unistra.fr/vizier/sed/
} 
Acknowledgements. Based on observations obtained at the European Southern Observatory, Paranal, Chile (ESO programme No. 0101.D-0399(B)). This paper includes data collected by the TESS mission. Funding for the TESS mission is provided by the NASA Explorer Program. The scientific results reported in this article are based in part on observations made by the Chandra X-ray Observatory under ObsID 17745 (PI Hubrig). We would like to thank the anonymous referee for useful suggestions to the manuscript and J. Federico González for fruitful discussions on the nature of the companion. This research has made use of the Jean-Marie Mariotti Center SearchCal service (available at http://www.jmmc.fr/searchcal) and the JSDC catalogue (available at http://www . jmmc . fr/catalogue_jsdc.htm) co-developed by FIZEAU and LAOG/IPAG, and of the CDS Astronomical Databases SIMBAD and VIZIER.

\section{References}

Babel, J., \& Montmerle, T. 1997, A\&A, 323, 121

Bailey, J. D., Grunhut, J., \& Landstreet, J. D. 2015, A\&A, 575, A115

Carrier, F., North, P., Udry, S., \& Babel, J. 2002, A\&A, 394, 151

Chelli, A., Duvert, G., Bourgès, L., et al. 2016, A\&A, 589, A112

Ducati, J. R., Bevilacqua, C. M., Rembold, S. B., \& Ribeiro, D. 2001, ApJ, 558, 309

Eker, Z., Soydugan, F., Bilir, S., et al. 2020, MNRAS, 496, 3887

ESA 1997, The Hipparcos and Tycho Catalogues, ESA SP, 1200

Ferrario, L., Pringle, J. E., Tout, C. A., \& Wickramasinghe, D. T. 2009, MNRAS, 400, L71

Gaia Collaboration (Brown, A. G. A., et al.) 2018, A\&A, 616, A1
Gallenne, A., Mérand, A., Kervella, P., et al. 2015, A\&A, 579, A68

Grevesse, N., \& Sauval, A. J. 1998, Space Sci. Rev., 85, 161

Güdel, M. 2004, A\&ARv, 12, 71

Hensberge, H. 1993, in IAU Colloq. 138: Peculiar Versus Normal Phenomena in A-type and Related Stars, eds. M. M. Dworetsky, F. Castelli, \& R. Faraggiana, ASP Conf. Ser., 44, 547

Lafrasse, S., Mella, G., Bonneau, D., et al. 2010, Proc. SPIE, 7734, 77344E

Le Bouquin, J.-B., Berger, J.-P., Lazareff, B., et al. 2011, A\&A, 535, A67

Leto, P., Trigilio, C., Oskinova, L., et al. 2017, MNRAS, 467, 2820

Mathys, G. 2017, A\&A, 601, A14

Mathys, G., Hubrig, S., Landstreet, J. D., Lanz, T., \& Manfroid, J. 1997, A\&AS, 123,353

Mozurkewich, D., Armstrong, J. T., \& Hindsley, R. B. 2003, AJ, 126, 2502

Oskinova, L. M., Ignace, R., Leto, P., \& Postnov, K. A. 2020, A\&A, 641, L8

Renson, P., \& Manfroid, J. 2009, A\&A, 498, 961

Robrade, J., Oskinova, L. M., Schmitt, J. H. M. M., et al. 2018, A\&A, 619, A33

Sana, H., de Mink, S. E., de Koter, A., et al. 2012, Science, 337, 444

Schmidt-Kaler, T. 1982 Landolt-Börnstein - VI/2B (Heidelberg, Berlin: Springer), 1

Schöller, M. 2007, New Astron. Rev., 51, 628

Slettebak, A., Collins, G. W., II, Boyce, P. B., White, N. M., \& Parkinson, T. D. 1975, ApJS, 29, 137

Smith, R. K., Brickhouse, N. S., Liedahl, D. A., \& Raymond, J. C. 2001, ApJ, 556, L91

Stelzer, B., Robrade, J., Schmitt, J. H. M. M., \& Bouvier, J. 2009, A\&A, 493, 3

Stibbs, D. W. N. 1950, MNRAS, 110, 395

Tutukov, A. V., \& Fedorova, A. V. 2010, Astron. Rep., 54, 156 